\title{
Social determinants of health associated with severity and mortality in patients with COVID-19
}

\author{
Rodrigo Núñez-Cortés, ${ }^{1 *}$ Maritza Ortega-Palavecinos, ${ }^{2}$ Camilo Soto-Carmona, ${ }^{3}$ Pablo Torres-Gangas, ${ }^{3}$ \\ Ma. Paz Concha-Rivero ${ }^{4}$ and Rodrigo Torres-Castro ${ }^{1}$ \\ ${ }^{1}$ Department of Physical Therapy, Faculty of Medicine, Universidad de Chile; ${ }^{2}$ Social Services, Hospital Clínico La Florida; ${ }^{3}$ Department of Physical \\ Therapy, Hospital Clínico La Florida; ${ }^{4}$ Nursing Department, Hospital Clínico La Florida. Santiago, Chile
}

\begin{abstract}
Introduction: Historically, pandemics have resulted in higher mortality rates in the most vulnerable populations. Social determinants of health $(S D H)$ have been associated with people morbidity and mortality at different levels. Objective: To determine the relationship between SDH and COVID-19 severity and mortality. Methods: Retrospective study, where data from patients with COVID-19 were collected at a public hospital in Chile. Sociodemographic variables related to structural SDH were classified according to the following categories: gender, age (<65 years, $\geq 65$ years), secondary education (completed or not), work status (active, inactive) and income (<USD 320, $\geq$ USD 320). Results: A total of 1,012 laboratory-confirmed COVID-19 cases were included. Average age was $64.2 \pm 17.5$ years. Mortality of the entire sample was $14.5 \%$. Age, level of education, unemployment and income had a strong association with mortality $(p<0.001)$. Conclusions: The findings reinforce the idea that SDH should be considered a public health priority, which is why political efforts should focus on reducing health inequalities for future generations.
\end{abstract}

KEY WORDS: Social determinants of health. Severe acute respiratory syndrome. Coronavirus. Mortality.

\section{Determinantes sociales de la salud asociados a la severidad y mortalidad en pacientes con COVID-19}

\section{Resumen}

Introducción: Históricamente, las pandemias han tenido como resultado tasas de mortalidad más altas en las poblaciones más vulnerables. Los determinantes sociales de la salud (DSS) se han asociado a la morbimortalidad de las personas en diferentes niveles. Objetivo: Determinar la relación entre los DSS, la severidad de COVID-19 y la mortalidad por esta enfermedad. Métodos: Estudio retrospectivo en el que se recolectaron datos de pacientes con COVID-19 en un hospital público de Chile. Las variables sociodemográficas relacionadas con los DSS estructurales se clasificaron según las siguientes categorías: sexo, edad (< 65 años, $\geq 65$ años), educación secundaria (completada o no), condición de trabajo (activo, inactivo) e ingreso económico (< USD 320, $\geq$ USD 320). Resultados: Fueron incluidos 1012 casos con COVID-19 confirmados por laboratorio. La edad promedio fue de $64.2 \pm 17.5$ años. La mortalidad de la muestra total fue de $14.5 \%$. La edad, nivel educativo, desempleo e ingresos tuvieron fuerte asociación con la mortalidad $(p<0.001)$. Conclusiones: Los hallazgos refuerzan la idea de que los DSS deben considerarse una prioridad de salud pública, por lo que los esfuerzos políticos deben centrarse en reducir las desigualdades en salud para las generaciones futuras.

PALABRAS CLAVE: Determinantes sociales de la salud. Síndrome respiratorio agudo grave. Coronavirus. Mortalidad.

Correspondence:

${ }^{*}$ Rodrigo Núñez-Cortés

E-mail: r_nunez@uchile.cl
Gac Med Mex. 2021;157:263-270

Contents available at PubMed

www.gacetamedicademexico.com

0016-3813/@ 2020 Academia Nacional de Medicina de México, A.C.. Published by Permanyer. This is an open access article under the CC BY-NC-ND license (http://creativecommons.org/licenses/by-nc-nd/4.0/). 


\section{Introduction}

In December 2019, an outbreak of pneumonia of unknown etiology occurred in Wuhan, China, which resulted in the isolation of a new coronavirus strain. The World Health Organization named this virus severe acute respiratory syndrome coronavirus 2 (SARSCoV-2), ${ }^{1}$ which evolved into a pandemic in a few weeks. ${ }^{2}$ Coronavirus disease 2019 (COVID-19) clinical presentation mainly comprises fever, cough, dyspnea and lung parenchyma compromise, which, in some cases, progresses to severe acute respiratory syndrome, requires admission to intensive care units (ICUs) and, eventually, causes death. ${ }^{3}$

Patients are classified with severe or non-severe COVID-19 according to the guidelines established by the American Thoracic Society and the Infectious Diseases Society of America. ${ }^{4}$ Criteria for ICU admission include invasive mechanical ventilation or septic shock that makes administration of vasopressors necessary. ${ }^{5}$ Although there are known risk factors for disease worsening (e.g., comorbidities), ${ }^{1}$ numerous authors have proposed the need for social determinants implication to be considered in this big challenge for public health. ${ }^{6,7}$

Social determinants of health (SDH) are the conditions in which people are born, grow, work, live and age, including the broader set of forces and systems that influence on the conditions of daily living, ${ }^{8}$ relationships with the political system, social norms and environmental considerations..$^{9,10} \mathrm{SDH}$ have been associated with morbidity and mortality at different levels. ${ }^{11}$ For example, mortality in older adults is associated with economic difficulties, level of education, social participation, physical activity and smoking, among others..$^{12}$ Socioeconomic level, represented by the level of education, income and occupation,,$^{13}$ is also associated with higher mortality and lower life expectancy in different countries and ethnic groups. ${ }^{13,14}$ In addition, SDHs have played an important role in the emergence and spread of other pandemics, such as that caused by the influenza virus. ${ }^{15}$

Approximately $15-20 \%$ of patients with COVID-19 develop the severe stage of the disease and require hospitalization. ${ }^{16}$ This group particularly includes older adults and people with comorbidities. There is a strong association between severity, mortality of the disease and cardio-metabolic comorbidities such as obesity, diabetes or hypertension. ${ }^{17}$ These diseases are influenced by multifactorial causes (individual, social and environmental factors). Although some biological factors are essential for the development of these diseases, cultural and social components, together with socioeconomic factors such as profession and income, contribute to the development of social behaviors that are also crucial to their appearance. ${ }^{18}$

Historically, pandemics cause higher mortality rates in more vulnerable populations (e.g., the 1918-1919 influenza pandemic). ${ }^{19}$ With the COVID-19 pandemic, it is essential for the social factors that determine a poor prognosis to be understood and this way developing early interventions and control strategies. The purpose of this study was to determine the relationship between SDHs and the level of severity and mortality caused by COVID-19. A secondary objective was to identify the relationship between underlying comorbidities and COVID-19 mortality and severity.

\section{Methods}

In this retrospective study, data from patients with COVID-19 were collected in a public hospital of Chile; medical history of all laboratory-confirmed cases was reviewed at La Florida Clinical Hospital (Santiago, Chile) between April 1 and June 10, 2020 (10 weeks). Inclusion criteria were the following: age $\geq 18$ years, outpatients and hospitalized patients with a PCR test positive result using nasal and pharyngeal samples. ${ }^{20}$ There were no exclusion criteria. The study was approved by the ethics committee of the institution. The research was carried out in accordance with the ethical principles of the Declaration of Helsinki. Written informed consent was waived due to the retrospective nature of the study and the urgent need for data on COVID-19 to be collected.

All six researchers of this study agreed on the design a data-extraction form. Collected data included medical history, history of exposure, and underlying comorbidities. The study main variable was mortality and severity. Severe cases were defined as those that were admitted to the ICU due to respiratory failure requiring mechanical ventilation or due to septic shock that made vasopressor administration necessary. ${ }^{5}$ Sociodemographic variables related to structural SDHs were classified according to the following categories: gender (male, female), age ( $<65$ years, $\geq 65$ years), secondary education (completed or not), work status (active, inactive) and income ( $<$ USD 320, $\geq$ USD 320). Other SDHs that were considered were disability status (mental, intellectual or sensory) and the institutionalized adult category. Clinical outcomes were 
monitored until July 18, 2020, which was follow-up conclusion date (six weeks after the last patient was admitted).

All statistical analyses were carried out using SPSS, version 22.0 (IBM Corporation, Armonk, New York). Normality of data was verified using the Shapiro-Wilk test. Means and standard deviations were calculated for quantitative variables, and percentages for categorical variables. The chi-square test was used to compare categorical variables. The association between mortality and COVID-19 severity and SDHs was determined by means of the odds ratio (OR), with a $95 \%$ confidence interval $(\mathrm{Cl})$. Statistical significance was established at $p<0.05$. Effect sizes were characterized as small, moderate or large and were established by ORs of $1.68,3.47$ and 6.71 , respectively. ${ }^{21}$

\section{Results}

In this study, 1,012 laboratory-confirmed COVID-19 cases were included. Average age was $64.2 \pm 17.5$ years. A total of 364 patients $(36 \%)$ were hospitalized, and 70 patients (6.9\%) were admitted to the ICU. Total sample mortality was $14.5 \%$, while mortality of hospitalized cases was $39.3 \%$. Table 1 describes the baseline characterization of the entire sample.

A significant association was found between SDHs and COVID-19 mortality and severity. Regarding mortality, a large effect of age $\geq 65$ years (OR $=24,95 \%$ $\mathrm{Cl}=14.7-39.2, \mathrm{p}<0.001)$, level of education $(\mathrm{OR}=9.6$, $95 \% \mathrm{Cl}=4.6-20.3, \mathrm{p}<0.001)$, inactive work status (OR $=16.3,95 \% \mathrm{Cl}=9.8-27.3, \mathrm{p}<0.001)$ and lower income (OR $=8.5,95 \% \mathrm{Cl}=5.1-14.4, \mathrm{p}<0.001)$ was observed. An age-adjusted analysis maintained a significant association with inactive work status $(\mathrm{OR}=6.1$, $95 \% \mathrm{Cl}=2.2-16.8, \mathrm{p}<0.001)$ and lower income $(\mathrm{OR}=6.8,95 \% \mathrm{Cl}=1.9-23.9, \mathrm{p}=0.001)$. Disability status also showed a large effect associated with mortality $(\mathrm{OR}=8.3,95 \% \mathrm{Cl}=4.7-14.4, \mathrm{p}<0.001)$. As for severity, a large effect size of the level of education was observed $(\mathrm{OR}=9.4,95 \% \mathrm{Cl}=4.4-20.3$, $\mathrm{p}<0.001$ ).

The most prevalent comorbidities in deceased patients were hypertension (78.9\%), diabetes (44.9\%) and cardiovascular diseases (24.5\%). A large effect size was observed for hypertension ( $\mathrm{OR}=10.7,95 \%$ $\mathrm{Cl}=7.0-16.4, \mathrm{p}=0.001)$ and cerebrovascular diseases $(\mathrm{OR}=8.2,95 \% \mathrm{Cl}=4.5-15, \mathrm{p}=0.001)$. Table $2 \mathrm{de}$ tails the association between SDHs, comorbidities,
Table 1. Total sample baseline characteristics $(n=1,012)$

\begin{tabular}{l|c|c|}
\hline Age, mean \pm SD & \multicolumn{2}{|c|}{$64.2 \pm 17.5$} \\
\hline $\begin{array}{l}\text { Age group } \\
\quad 65 \text { years } \\
<65 \text { years }\end{array}$ & 299 & 29.5 \\
\hline Gender & 713 & 70.5 \\
$\quad$ Males & & \\
\hline Females & 439 & 43.4 \\
\hline Hospitalized & 573 & 56.6 \\
\hline Deceased & 364 & 36 \\
\hline ICU admission & 147 & 14.5 \\
\hline Invasive ventilation & 70 & 6.9 \\
\hline Tracheostomy & 86 & 8.5 \\
\hline
\end{tabular}

$\mathrm{SD}=$ standard deviation, $\mathrm{ICU}=$ intensive care unit.

and mortality, and table 3 , the association between $\mathrm{SDH}$, comorbidities, and disease severity.

\section{Discussion}

The purpose of this study was to determine the relationship between SDHs and COVID-19 mortality and severity. In our sample, we observed a mortality rate of $14.5 \%$ in all patients, and of $39.3 \%$ in hospitalized cases, which is much higher than that reported in other studies. ${ }^{22,23}$ Structural SDHs showed a strong association with mortality, including age, level of education, unemployment and income. These SDHs also showed a relationship with COVID-19 severity; $6.9 \%$ of the patients were admitted to the ICU.

As for age, adults aged $\geq 65$ years had a 24 -fold higher risk of mortality in comparison with younger subjects; this association was much stronger than that reported by other authors. ${ }^{24,25}$ These differences can be explained by the relationship between $\mathrm{SDH}$ and mortality in older adults at different levels, from lifestyles to socioeconomic macro-determinants (economic difficulties, level of education). For example, evidence shows that older adults constitute the most sedentary age group; ${ }^{26}$ in turn, sedentary lifestyle is associated with a mortality increase, development of chronic disease ${ }^{27}$ and an increase in other risk factors that impact COVID-19 level of severity (e.g., hypertension). ${ }^{25}$ In addition, income and level of education are factors that are also associated with higher mortality in older people,,$^{13}$ which is consistent with our findings. Another group susceptible to die 
Gaceta Médica de México. 2021;157

Table 2. Social determinants of health and comorbidities of patients with COVID-19 grouped according to clinical outcome

\begin{tabular}{|c|c|c|c|c|c|c|}
\hline \multirow[t]{2}{*}{ Characteristics } & \multicolumn{2}{|c|}{$\begin{array}{l}\text { Deceased } \\
(n=147)\end{array}$} & \multicolumn{2}{|c|}{$\begin{array}{l}\text { Not deceased } \\
(n=865)\end{array}$} & \multirow[t]{2}{*}{ OR (95 \% Cl) } & \multirow[t]{2}{*}{ p } \\
\hline & $\mathrm{n}$ & $\%$ & $n$ & $\%$ & & \\
\hline $\begin{array}{l}\text { Gender } \\
\text { Males } \\
\text { Females }\end{array}$ & $\begin{array}{l}80 \\
67\end{array}$ & $\begin{array}{l}54.4 \\
45.6\end{array}$ & $\begin{array}{l}359 \\
506\end{array}$ & $\begin{array}{l}41.5 \\
58.5\end{array}$ & $1.68(1.18-2.39)$ & 0.002 \\
\hline $\begin{array}{l}\text { Age } \\
\geq 65 \text { years } \\
<65 \text { years }\end{array}$ & $\begin{array}{c}126 \\
21\end{array}$ & $\begin{array}{l}85.7 \\
14.3\end{array}$ & $\begin{array}{l}173 \\
692\end{array}$ & $\begin{array}{l}20 \\
80\end{array}$ & 24 (14.68-39.22) & $<0.001$ \\
\hline $\begin{array}{l}\text { Secondary education } \\
\text { Incomplete } \\
\text { Complete } \\
\text { Unknown }\end{array}$ & $\begin{array}{l}90 \\
18 \\
39\end{array}$ & $\begin{array}{l}61.2 \\
12.2 \\
26.5\end{array}$ & $\begin{array}{c}272 \\
524 \\
69\end{array}$ & $\begin{array}{c}31.4 \\
60.6 \\
8\end{array}$ & $9.63(4.57-20.29)$ & $<0.001$ \\
\hline $\begin{array}{l}\text { Work status } \\
\text { Inactive } \\
\text { Active }\end{array}$ & $\begin{array}{c}129 \\
18\end{array}$ & $\begin{array}{l}87.8 \\
12.2\end{array}$ & $\begin{array}{l}264 \\
601\end{array}$ & $\begin{array}{l}30.5 \\
69.5\end{array}$ & $16.32(9.75-27.28)$ & $<0.001$ \\
\hline $\begin{array}{l}\text { Income } \\
\quad<\text { USD } 320 \\
\geq \text { USD } 320\end{array}$ & $\begin{array}{c}130 \\
17\end{array}$ & $\begin{array}{l}88.4 \\
11.6\end{array}$ & $\begin{array}{l}409 \\
456\end{array}$ & $\begin{array}{l}47.3 \\
52.7\end{array}$ & $8.5(5.05-14.38)$ & $<0.001$ \\
\hline $\begin{array}{l}\text { Institutionalized adults } \\
\text { Yes } \\
\text { No }\end{array}$ & $\begin{array}{c}13 \\
134\end{array}$ & $\begin{array}{c}8.8 \\
91.2\end{array}$ & $\begin{array}{l}21 \\
844\end{array}$ & $\begin{array}{c}2.4 \\
97.6\end{array}$ & $3.89(1.91-7.97)$ & $<0.001$ \\
\hline $\begin{array}{l}\text { Disability situation } \\
\text { Yes } \\
\text { No }\end{array}$ & $\begin{array}{c}31 \\
116\end{array}$ & $\begin{array}{l}21.1 \\
78.9\end{array}$ & $\begin{array}{l}27 \\
838\end{array}$ & $\begin{array}{c}3.1 \\
96.9\end{array}$ & $8.29(4.77-14.39)$ & $<0.001$ \\
\hline $\begin{array}{l}\text { History of smoking } \\
\text { Yes } \\
\text { No }\end{array}$ & $\begin{array}{c}9 \\
138\end{array}$ & $\begin{array}{c}6.1 \\
93.9\end{array}$ & $\begin{array}{c}89 \\
776\end{array}$ & $\begin{array}{l}10.3 \\
89.7\end{array}$ & $0.569(0.28-1.16)$ & 0.071 \\
\hline $\begin{array}{l}\text { Alcoholism } \\
\text { Yes } \\
\text { No }\end{array}$ & $\begin{array}{c}7 \\
140\end{array}$ & $\begin{array}{c}4.8 \\
95.2\end{array}$ & $\begin{array}{c}45 \\
820\end{array}$ & $\begin{array}{c}5.2 \\
94.8\end{array}$ & $0.91(0.4-2.06)$ & 0.509 \\
\hline $\begin{array}{l}\text { Obesity } \\
\text { Yes } \\
\text { No }\end{array}$ & $\begin{array}{c}19 \\
128\end{array}$ & $\begin{array}{l}12.9 \\
87.1\end{array}$ & $\begin{array}{c}79 \\
786\end{array}$ & $\begin{array}{c}9.1 \\
90.1\end{array}$ & $1.47(0.86-2.52)$ & 0.102 \\
\hline $\begin{array}{l}\text { Hypertension } \\
\text { Yes } \\
\text { No }\end{array}$ & $\begin{array}{c}116 \\
31\end{array}$ & $\begin{array}{l}78.9 \\
21.1\end{array}$ & $\begin{array}{l}224 \\
641\end{array}$ & $\begin{array}{l}25.9 \\
74.1\end{array}$ & $10.71(7.0-16.37)$ & $<0.001$ \\
\hline $\begin{array}{l}\text { Diabetes } \\
\text { Yes } \\
\text { No }\end{array}$ & $\begin{array}{l}66 \\
81\end{array}$ & $\begin{array}{l}44.9 \\
55.1\end{array}$ & $\begin{array}{l}137 \\
728\end{array}$ & $\begin{array}{l}15.8 \\
84.2\end{array}$ & $4.33(2.98-6.28)$ & $<0.001$ \\
\hline $\begin{array}{l}\text { Cardiovascular disease } \\
\text { Yes } \\
\text { No }\end{array}$ & $\begin{array}{c}36 \\
111\end{array}$ & $\begin{array}{l}24.5 \\
75.5\end{array}$ & $\begin{array}{c}53 \\
812\end{array}$ & $\begin{array}{c}6.1 \\
93.9\end{array}$ & $4.96(3.11-7.93)$ & $<0.001$ \\
\hline $\begin{array}{l}\text { Cerebrovascular disease } \\
\text { Yes } \\
\text { No }\end{array}$ & $\begin{array}{c}26 \\
121\end{array}$ & $\begin{array}{l}17.7 \\
82.3\end{array}$ & $\begin{array}{c}22 \\
843\end{array}$ & $\begin{array}{c}2.5 \\
95.5\end{array}$ & $8.23(4.52-14.98)$ & $<0.001$ \\
\hline $\begin{array}{l}\text { COPD } \\
\text { Yes } \\
\text { No }\end{array}$ & $\begin{array}{c}17 \\
130\end{array}$ & $\begin{array}{l}11.6 \\
88.4\end{array}$ & $\begin{array}{c}23 \\
842\end{array}$ & $\begin{array}{c}2.7 \\
97.3\end{array}$ & $4.78(2.49-9.2)$ & $<0.001$ \\
\hline
\end{tabular}


Núñez-Cortés R, et al.: Social determinants and COVID-19

Table 2. Social determinants of health and comorbidities of patients with COVID-19 grouped according to clinical outcome (Continued)

\begin{tabular}{|c|c|c|c|c|c|c|}
\hline \multirow[t]{2}{*}{ Characteristics } & \multicolumn{2}{|c|}{$\begin{array}{l}\text { Deceased } \\
(n=147)\end{array}$} & \multicolumn{2}{|c|}{$\begin{array}{l}\text { Not deceased } \\
(n=865)\end{array}$} & \multirow[t]{2}{*}{ OR (95\% CI) } & \multirow[t]{2}{*}{$p$} \\
\hline & $\mathrm{n}$ & $\%$ & $\mathrm{n}$ & $\%$ & & \\
\hline $\begin{array}{l}\text { Other lung diseas } \\
\text { Yes } \\
\text { No }\end{array}$ & $\begin{array}{c}18 \\
129\end{array}$ & $\begin{array}{l}12.2 \\
87.8\end{array}$ & $\begin{array}{c}46 \\
819\end{array}$ & $\begin{array}{c}5.3 \\
94.7\end{array}$ & $2.48(1.39-4.41)$ & 0.03 \\
\hline $\begin{array}{l}\text { Chronic kidney di } \\
\text { Yes } \\
\text { No }\end{array}$ & $\begin{array}{c}21 \\
126\end{array}$ & $\begin{array}{l}14.3 \\
85.7\end{array}$ & $\begin{array}{c}29 \\
836\end{array}$ & $\begin{array}{c}3.4 \\
96.6\end{array}$ & $4.81(2.65-8.68)$ & $<0.001$ \\
\hline $\begin{array}{l}\text { Cancer } \\
\text { Yes } \\
\text { No }\end{array}$ & $\begin{array}{c}9 \\
138\end{array}$ & $\begin{array}{c}6.1 \\
93.9\end{array}$ & $\begin{array}{c}20 \\
845\end{array}$ & 97.72 .3 & $2.75(1.23-6.18)$ & 0.017 \\
\hline $\begin{array}{l}\text { Immunodeficienc } \\
\text { Yes } \\
\text { No }\end{array}$ & $\begin{array}{c}3 \\
144\end{array}$ & $\begin{array}{l}2.0 \\
98\end{array}$ & $\begin{array}{c}12 \\
853\end{array}$ & $\begin{array}{c}1.4 \\
98.6\end{array}$ & $1.48(0.41-5.31)$ & 0.375 \\
\hline
\end{tabular}

$\mathrm{COPD}=$ chronic obstructive pulmonary disease, $\mathrm{OR}=$ odds ratio, $\mathrm{Cl}=$ confidence interval.

Table 3. Social determinants of health and comorbidities of patients with COVID-19 grouped according to disease severity

\begin{tabular}{|c|c|c|c|c|c|c|}
\hline \multirow[t]{2}{*}{ Characteristics } & \multicolumn{2}{|c|}{$\begin{array}{l}\text { Severe } \\
(n=70)\end{array}$} & \multicolumn{2}{|c|}{$\begin{array}{c}\text { Non-severe } \\
(\mathrm{n}=942)\end{array}$} & \multirow[t]{2}{*}{ OR (95\% CI) } & \multirow[t]{2}{*}{$p$} \\
\hline & $n$ & $\%$ & $\mathrm{n}$ & $\%$ & & \\
\hline $\begin{array}{l}\text { Gender } \\
\text { Males } \\
\text { Females }\end{array}$ & $\begin{array}{l}41 \\
29\end{array}$ & $\begin{array}{l}58.6 \\
41.4\end{array}$ & $\begin{array}{l}398 \\
544\end{array}$ & $\begin{array}{l}42.3 \\
57.7\end{array}$ & $1.93(1.18-3.16)$ & 0.006 \\
\hline $\begin{array}{l}\text { Age } \\
\geq 65 \text { years } \\
<65 \text { years }\end{array}$ & $\begin{array}{l}44 \\
26\end{array}$ & $\begin{array}{l}62.9 \\
37.1\end{array}$ & $\begin{array}{l}255 \\
687\end{array}$ & $\begin{array}{l}27.1 \\
72.9\end{array}$ & $4.56(2.75-7.56)$ & $<0.001$ \\
\hline $\begin{array}{l}\text { Secondary educat } \\
\text { Incomplete } \\
\text { Complete } \\
\text { Unknown }\end{array}$ & $\begin{array}{c}44 \\
8 \\
18\end{array}$ & $\begin{array}{l}62.9 \\
11.4 \\
25.7\end{array}$ & $\begin{array}{c}314 \\
538 \\
90\end{array}$ & $\begin{array}{c}33.3 \\
57.1 \\
9.6\end{array}$ & $9.42(4.38-20.27)$ & $<0.001$ \\
\hline $\begin{array}{l}\text { Work status } \\
\text { Inactive } \\
\text { Active }\end{array}$ & $\begin{array}{l}37 \\
33\end{array}$ & $\begin{array}{l}52.9 \\
47.1\end{array}$ & $\begin{array}{l}356 \\
586\end{array}$ & $\begin{array}{l}37.8 \\
62.2\end{array}$ & $1.85(1.13-3.01)$ & 0.010 \\
\hline $\begin{array}{l}\text { Income } \\
<\text { < USD } 320 \\
\geq \text { USD } 320\end{array}$ & $\begin{array}{l}52 \\
18\end{array}$ & $\begin{array}{l}74.3 \\
25.7\end{array}$ & $\begin{array}{l}487 \\
455\end{array}$ & $\begin{array}{l}51.7 \\
48.3\end{array}$ & $2.69(1.56-4.68)$ & $<0.001$ \\
\hline $\begin{array}{l}\text { Institutionalized ad } \\
\text { Yes } \\
\text { No }\end{array}$ & $\begin{array}{c}2 \\
68\end{array}$ & $\begin{array}{c}2.9 \\
97.1\end{array}$ & $\begin{array}{c}32 \\
910\end{array}$ & $\begin{array}{c}3.4 \\
96.6\end{array}$ & $0.84(0.19-3.56)$ & 0.578 \\
\hline $\begin{array}{l}\text { Disability situation } \\
\text { Yes } \\
\text { No }\end{array}$ & $\begin{array}{c}4 \\
64\end{array}$ & $\begin{array}{c}5.7 \\
94.3\end{array}$ & $\begin{array}{c}54 \\
888\end{array}$ & $\begin{array}{c}5.7 \\
94.3\end{array}$ & $0.99(0.35-2.83)$ & 0.627 \\
\hline $\begin{array}{l}\text { History of smoking } \\
\text { Yes } \\
\text { No }\end{array}$ & $\begin{array}{c}6 \\
64\end{array}$ & $\begin{array}{c}8.6 \\
91.4\end{array}$ & $\begin{array}{c}92 \\
850\end{array}$ & $\begin{array}{c}9.8 \\
90.2\end{array}$ & $0.87(0.37-2.069$ & 0.473 \\
\hline $\begin{array}{l}\text { Alcoholism } \\
\text { Yes } \\
\text { No }\end{array}$ & $\begin{array}{c}7 \\
63\end{array}$ & $\begin{array}{l}10 \\
90\end{array}$ & $\begin{array}{c}45 \\
897\end{array}$ & $\begin{array}{c}4.8 \\
95.2\end{array}$ & $2.21(0.96-5.11)$ & 0.061 \\
\hline
\end{tabular}


Table 3. Social determinants of health and comorbidities of patients with COVID-19 grouped according to disease severity (Continued)

\begin{tabular}{|c|c|c|c|c|c|c|}
\hline \multirow[t]{2}{*}{ Characteristics } & \multicolumn{2}{|c|}{$\begin{array}{l}\text { Severe } \\
(n=70)\end{array}$} & \multicolumn{2}{|c|}{$\begin{array}{l}\text { Non-severe } \\
(n=942)\end{array}$} & \multirow[t]{2}{*}{ OR (95\% Cl) } & \multirow[t]{2}{*}{$p$} \\
\hline & $n$ & $\%$ & $n$ & $\%$ & & \\
\hline $\begin{array}{l}\text { Obesity } \\
\text { Yes } \\
\text { No }\end{array}$ & $\begin{array}{l}15 \\
55\end{array}$ & $\begin{array}{l}21.4 \\
78.6\end{array}$ & $\begin{array}{c}83 \\
859\end{array}$ & $\begin{array}{c}8.8 \\
91.2\end{array}$ & $2.82(1.53-5.21)$ & 0.002 \\
\hline $\begin{array}{l}\text { Hypertension } \\
\text { Yes } \\
\text { No }\end{array}$ & $\begin{array}{l}52 \\
18\end{array}$ & $\begin{array}{l}74.3 \\
25.7\end{array}$ & $\begin{array}{l}288 \\
654\end{array}$ & $\begin{array}{l}30.6 \\
69.4\end{array}$ & $6.56(3.77-11.41)$ & $<0.001$ \\
\hline $\begin{array}{l}\text { Diabetes } \\
\text { Yes } \\
\text { No }\end{array}$ & $\begin{array}{l}30 \\
40\end{array}$ & $\begin{array}{l}42.9 \\
57.1\end{array}$ & $\begin{array}{l}173 \\
769\end{array}$ & $\begin{array}{l}18.4 \\
81.6\end{array}$ & $3.33(2.02-5.5)$ & $<0.001$ \\
\hline $\begin{array}{l}\text { Cardiovascular d } \\
\text { Yes } \\
\text { No }\end{array}$ & $\begin{array}{l}12 \\
58\end{array}$ & $\begin{array}{l}17.1 \\
82.9\end{array}$ & $\begin{array}{c}77 \\
865\end{array}$ & $\begin{array}{c}8.2 \\
91.8\end{array}$ & $2.32(1.19-4.51)$ & 0.015 \\
\hline $\begin{array}{l}\text { Cerebrovascular } \\
\text { Yes } \\
\text { No }\end{array}$ & $\begin{array}{c}6 \\
64\end{array}$ & $\begin{array}{c}8.6 \\
91.4\end{array}$ & $\begin{array}{c}42 \\
900\end{array}$ & $\begin{array}{c}4.5 \\
95.5\end{array}$ & $2.01(0.82-4.9)$ & 0.107 \\
\hline $\begin{array}{l}\text { COPD } \\
\text { Yes } \\
\text { No }\end{array}$ & $\begin{array}{c}5 \\
65\end{array}$ & $\begin{array}{c}7.1 \\
92.9\end{array}$ & $\begin{array}{c}35 \\
907\end{array}$ & $\begin{array}{c}3.7 \\
96.3\end{array}$ & $1.99(0.76-5.3)$ & 0.136 \\
\hline $\begin{array}{l}\text { Other lung diseas } \\
\text { Yes } \\
\text { No }\end{array}$ & $\begin{array}{c}7 \\
63\end{array}$ & $\begin{array}{l}10 \\
90\end{array}$ & $\begin{array}{c}57 \\
885\end{array}$ & $\begin{array}{c}6.1 \\
93.9\end{array}$ & $1.73(0.76-3.93)$ & 0.146 \\
\hline $\begin{array}{l}\text { Chronic kidney di } \\
\text { Yes } \\
\text { No }\end{array}$ & $\begin{array}{c}7 \\
63\end{array}$ & $\begin{array}{l}10 \\
90\end{array}$ & $\begin{array}{c}43 \\
899\end{array}$ & $\begin{array}{c}4.6 \\
95.4\end{array}$ & $2.32(1.0-5.37)$ & 0.051 \\
\hline $\begin{array}{l}\text { Cancer } \\
\text { Yes } \\
\text { No }\end{array}$ & $\begin{array}{c}2 \\
68\end{array}$ & $\begin{array}{l}2.9 \\
97.1\end{array}$ & $\begin{array}{c}27 \\
915\end{array}$ & $\begin{array}{l}2.9 \\
97.1\end{array}$ & $0.99(0.23-4.28)$ & 0.675 \\
\hline $\begin{array}{l}\text { Immunodeficienci } \\
\text { Yes } \\
\text { No }\end{array}$ & $\begin{array}{c}2 \\
68\end{array}$ & $\begin{array}{l}2.9 \\
97.1\end{array}$ & $\begin{array}{c}13 \\
929\end{array}$ & $\begin{array}{c}1.4 \\
98.6\end{array}$ & $2.1(0.47-9.5)$ & 0.278 \\
\hline
\end{tabular}

$\mathrm{COPD}=$ chronic obstructive pulmonary disease, $\mathrm{OR}=$ odds ratio, $\mathrm{Cl}=$ confidence interval.

from COVID-19 are institutionalized adults. The population that resides in long-term care facilities generally corresponds to older adults suffering from multiple diseases. ${ }^{28}$

As for socioeconomic level, our results are similar to those reported by other authors. Ramírez and $L^{29}$ found that SDHs such as poverty and unemployment were positively and significantly associated with a higher COVID-19 case fatality rate in older people. These findings are explained by a higher prevalence of poverty and unemployment according to different geographical areas. Chile has been identified as one of the countries with the highest income inequality, according to the Organization for Economic Co-operation and Development, ${ }^{30}$ where economic segregation (expressed by income) is associated with higher mortality rates. ${ }^{31}$ These findings reinforce the idea of considering SDHs as a priority for public health, with political efforts aimed at reducing health inequity for future generations.

Regarding people with disabilities, in this group we observed a higher association with mortality. People on disability situation are more likely to obtain lower-than-expected health outcomes, particularly in low- and middle-income countries. ${ }^{32,33}$ Disability is related to other SDHs (such as unemployment $)^{34}$ and other health conditions that increase COVID-19 mortality rate as, for example, diabetes and cardiovascular disease..$^{33}$ Our findings show the urgent need for the provision health care to this group to be improved. 
Regarding comorbidities, hypertension was a frequently reported comorbidity in our population, with a significant association with COVID-19 mortality and severity, similar to that reported by other authors. ${ }^{1,35}$ Although comorbidities associated with risk for COVID-19 worsening or death have been widely described in the literature, people with a lower socioeconomic level have these risk factors more often, including hypertension, diabetes, obesity, heart conditions and chronic respiratory diseases. ${ }^{6,36}$ Therefore, we cannot discard that the strength of association between these variables is also related to SDHs in our population. For example, hypertension is the condition responsible for the greatest burden of disease in Chile, and it affects a larger proportion of people with low levels education and older adults. ${ }^{37}$ This public health problem in Chile has become more evident with the pandemic, which shows the importance of generating public health policies able to prevent the prevalence of these risk factors.

Regarding COVID-19 survivors future needs, it should be noted that limitations for practicing exercise, together with physical and psychological sequelae, are the main consequences of severe lung damage, ${ }^{38}$ and that a significant group of these patients will therefore require rehabilitation. ${ }^{39} \mathrm{SDH}$ are also related to access to and participation in rehabilitation programs. ${ }^{40}$ For example, economic difficulties comprise a structural barrier that affects adherence to pulmonary rehabilitation. ${ }^{41}$ Multidisciplinary rehabilitation teams should be aware of the health inequalities caused by SDHs and should implement specialized strategies to improve long-term functionality of these patients.

One limitation of our study was the possible underreporting of comorbidities on each patient medical history, which could underestimate the true strength of their association with clinical outcomes. However, our findings were consistent with those reported in the literature. It is also important noting that they apply only to the reality of a country and, therefore, multicenter investigations are required in order for these findings to be confirmed.

\section{Conclusions}

Structural SDHs had a strong association with COVID-19 mortality and severity, particularly age, level of education, unemployment, and income. These findings are relevant because they confirm that socioeconomic conditions can influence both population health and people individual characteristics. It is important for
SDHs to be considered a public health priority and for political efforts to focus on reducing health inequalities for future generations.

\section{Conflict of interests}

The authors declare that they have no conflicts of interest.

\section{Funding}

This research did not receive any specific grants from agencies of public, commercial or non-profit sectors.

\section{Ethical disclosures}

Protection of human and animal subjects. The authors declare that the procedures that were followed adhered to the ethical standards of the responsible committee for experimentation on human beings and were in agreement with the World Medical Association and the Declaration of Helsinki.

Confidentiality of data. The authors declare that they followed the protocols of their work center on the publication of patient data.

Right to privacy and informed consent. The authors declare that no patient data appear in this article.

\section{References}

1. Zhou F, Yu T, Du R, Fan G, Liu Y, Xiang J, et al. Clinical course and risk factors for mortality of adult inpatients with COVID-19 in Wuhan, China: a retrospective cohort study. Lancet. 2020;395:1054-1062.

2. Adhikari SP, Meng S, Wu YJ, Mao YP, Ye RX, Wang QZ, et al. Epidemiology, causes, clinical manifestation and diagnosis, prevention and control of coronavirus disease (COVID-19) during the early outbreak period: a scoping review. Infect Dis Poverty. 2020;9:29.

3. Zhu N, Zhang D, Wang W, Li X, Yang B, Song J, et al. A novel coronavirus from patients with pneumonia in China, 2019. N Engl J Med. 2020;382:727-733.

4. Metlay JP, Waterer GW, Long AC, Anzueto A, Brozek J, Crothers K, et al. Diagnosis and treatment of adults with community-acquired pneumonia. An official clinical practice guideline of the American Thoracic Society and Infectious Diseases Society of America. Am J Respir Crit Care Med. 2019;200:e45-e67.

5. Gearhart AM, Furmanek S, English C, Ramírez J, Cavallazzi R. Predicting the need for ICU admission in community-acquired pneumonia. Respir Med. 2019;155:61-65.

6. Bambra C, Riordan R, Ford J, Matthews F. The COVID-19 pandemic and health inequalities. J Epidemiol Community Health. 2020;74:964-968.

7. Takian A, Kiani MM, Khanjankhani K. COVID-19 and the need to prioritize health equity and social determinants of health. Int J Public Health. 2020;65:521-523.

8. Marmot M, Friel S, Bell R, Houweling TAJ, Taylor S. Closing the gap in a generation: health equity through action on the social determinants of health. Lancet. 2008;372:1661-1669.

9. Marmot M. Social determinants of health inequalities. Lancet. 2005;365:1099-1104.

10. Hernández LJ, Ocampo J, Ríos DS, Calderón C. The WHO model as a guideline for public health based on social determinants. Rev Salud Publica (Bogota). 2017;19:393-395. 
11. Solar O, Irwin A. Social determinants, political contexts and civil society action: a historical perspective on the Commission on Social Determinants of Health. Health Promot J Austr. 2006;17:180-185.

12. De Lima-Silva V, Pessoa-Cesse EÂ, Pessoa-Militão-de Albuquerque MF. Social determinants of death among the elderly: a systematic literature review. Rev Bras Epidemiol. 2014;17:178-193.

13. Bassuk SS, Berkman LF, Amick BC. Socioeconomic status and mortality among the elderly: findings from four US communities. Am J Epidemiol. 2002;155:520-533

14. Signorello LB, Cohen SS, Williams DR, Munro HM, Hargreaves MK Blot WJ. Socioeconomic status, race, and mortality: a prospective cohort study. Am J Public Health. 2014;104:e98-e107.

15. Sooryanarain $\mathrm{H}$, Elankumaran $\mathrm{S}$. Environmental role in influenza virus outbreaks. Annu Rev Anim Biosci. 2015;3:347-373.

16. Berlin DA, Gulick RM, Martínez FJ. Severe Covid-19. N Engl J Med. 2020;383:2451-2460

17. Richardson S, Hirsch JS, Narasimhan M, Crawford JM, McGinn T, Davidson $\mathrm{KW}$, et al. Presenting characteristics, comorbidities, and outcomes among 5700 patients hospitalized with COVID-19 in the New York City area. JAMA. 2020;323:2052-2059.

18. Noriea AH, Patel FN, Werner DA, Peek ME. A narrative review of physician perspectives regarding the social and environmental determinants of obesity. Curr Diab Rep. 2018;18:24.

19. Summers JA, Stanley J, Baker MG, Wilson N. Risk factors for death from pandemic influenza in 1918-1919: a case-control study. Influenza Other Respir Viruses. 2014;8:329-338

20. Huang C, Wang Y, Li X, Ren L, Zhao J, Hu Y, et al. Clinical features of patients infected with 2019 novel coronavirus in Wuhan, China. Lancet. 2020;395:497-506.

21. Chen $\mathrm{H}$, Cohen $\mathrm{P}$, Chen $\mathrm{S}$. How big is a big odds ratio? Interpreting the magnitudes of odds ratios in epidemiological studies. Communications in Statistics - Simulation and Computation. 2010;39:860-864.

22. Epidemiology Working Group for NCIP Epidemic Response, Chinese Center for Disease Control and Prevention. The epidemiological characteristics of an outbreak of 2019 novel coronavirus diseases (COVID-19) in China. Zhonghua Liu Xing Bing Xue Za Zhi. 2020;41:145-151.

23. Chen N, Zhou M, Dong X, Qu J, Gong F, Han Y, et al. Epidemiological and clinical characteristics of 99 cases of 2019 novel coronavirus pneumonia in Wuhan, China: a descriptive study. Lancet. 2020;395:507-513.

24. Du RH, Liang LR, Yang CQ, Wang W, Cao TZ, Li M, et al. Predictors of mortality for patients with COVID-19 pneumonia caused by SARS-CoV-2: a prospective cohort study. Eur Respir J. 2020:55:2000524.

25. Li X, Xu S, Yu M, Wang K, Tao Y, Zhou Y, et al. Risk factors for severity and mortality in adult COVID-19 inpatients in Wuhan. J Allergy Clin Immunol. 2020;146:110-118.
26. Wullems JA, Verschueren SMP, Degens $\mathrm{H}$, Morse $\mathrm{Cl}$, Onambélé $\mathrm{GL}$. A review of the assessment and prevalence of sedentarism in older adults, its physiology/health impact and non-exercise mobility counter-measures. Biogerontology. 2016;17:547-565.

27. Tremblay MS, Colley RC, Saunders TJ, Healy GN, Owen N. Physiological and health implications of a sedentary lifestyle. Appl Physiol Nutr Metab. 2010;35:725-740.

28. D’Adamo H, Yoshikawa T, Ouslander JG. Coronavirus disease 2019 in geriatrics and long-term care: the ABCDs of COVID 019. J Am Geriatr Soc. 2020;68:912-917

29. Ramírez IJ, Lee J. COVID-19 emergence and social and health determinants in Colorado: a rapid spatial analysis. Int $\mathrm{J}$ Environ Res Public Health. 2020;17:3856.

30. Organisation for Economic Co-operation and Development [Internet]. France: Society at a Glance 2014; 2014.

31. Sánchez H, Albala C. Inequalities in health. Adult mortality in communities of Metropolitan Santiago. Rev Med Chil. 2004;132:453-460.

32. Armitage R, Nellums LB. The COVID-19 response must be disability inclusive. Lancet Public Health. 2020;5:e257.

33. The Lancet. Prioritising disability in universal health coverage. Lancet. 2019;394:187.

34. Bambra C, Pope D. What are the effects of anti-discriminatory legislation on socioeconomic inequalities in the employment consequences of ill health and disability? J Epidemiol Community Health. 2007;61:421-426.

35. Guan WJ, Liang WH, Zhao Y, Liang HR, Chen ZS, Li YM, et al. Comorbidity and its impact on 1590 patients with COVID-19 in China: a nationwide analysis. Eur Respir J. 2020;55:2000547.

36. Guo L, Wei D, Zhang X, Wu Y, Li Q, Zhou M, et al. Clinical features predicting mortality risk in patients with viral pneumonia: the MuLBSTA score. Front Microbiol. 2019;10:2752.

37. Ministerio de Salud. Encuesta Nacional de Salud 2009-2010. Chile: Ministerio de Salud; 2010

38. Herridge MS, Tansey CM, Matté A, Tomlinson G, Díaz-Granados N, Cooper A, et al. Functional disability 5 years after acute respiratory distress syndrome. N Engl J Med. 2011;364:1293-1304.

39. Rivera-Lillo G, Torres-Castro R, Fregonezi G, Vilaró J, Puppo H. Challenge for rehabilitation after hospitalization for COVID-19. Arch Phys Med Rehabil. 2020;101:1470-1471.

40. Frier A, Barnett F, Devine S. The relationship between social determinants of health, and rehabilitation of neurological conditions: a systematic literature review. Disabil Rehabil. 2017;39:941-948.

41. Oates GR, Niranjan SJ, Ott C, Scarinci I, Schumann C, Parekh T, et al. Adherence to pulmonary rehabilitation in COPD: A qualitative exploration of patient perspectives on barriers and facilitators. J Cardiopulm Rehabil Prev. 2019;39:344-349. 